\title{
Perioperative Care for Organ Transplant Recipient: Time for Paradigm Shift
}

\author{
Alexander A. Vitin
}

Department of Anesthesiology \& Pain Medicine, University of Washington Medical Center, Seattle WA, USA

Transplantation medicine, one of the emerging major medical disciplines, encompasses a wide variety of clinical subspecialties.

The concept of replacing organs which are failing or showing insufficiency, with single or multiple organs, either artificial or from donors, is accepted in literally every clinical field

There is explosive growth in the transplant sector driven by an ever-increasing patient demand fuelled by the already well-proven efficiency of organ transplantation as an ultimate treatment for end-stage organs failure and the ever-expanding infrastructure of the transplantation industry.

The foundation of this industry rests on two pillars: transplantation medicine and transplantation science. The sheer magnitude of the progress within the transplantation industry, as it stands today, maybe best illustrated by impressive statistics and facts, accomplishments and ongoing research trends.

Today, organ and tissue transplantation operations are being performed in more than one hundred and eleven countries, encompassing about $81 \%$ of the world's population. New countries are joining this club every year. Close to 140,000 organs are being transplanted every year worldwide. According to the most recently published OPTN data, in the USA alone, from January 1, 1988, to April 30, 2019, 451,847 kidney, 166,383 liver, 73,216 heart 38,989 lung, 23,959 kidneypancreas and numerous other organ transplantations have been performed in the more than eighty transplant programs. The current trend indicates an exponential increase in these numbers $[1,2]$.

Fifteen international and more than 140 national organizations worldwide are ceaselessly promoting and coordinating research outcomes as well as implementing, developing and improving all practical aspects of organ donation and transplantation procedures.
Ever since the very first successful solid organ transplants, transplantation-related science has exhibited an exponential growth. Physicians and researchers from many specialities are getting more involved in transplantation medicine, which has outgrown the boundaries of any one speciality and must now be considered a whole new field of medical science in its own right.

Results of clinical and experimental research provide imposing and useful data for publication in a myriad of specialist publications worldwide. There are more than seventy-five periodic issues, among which more than forty high-impact journals which publish results of research. A PubMed search returned about 800,000 titles of the indexed publications pertinent to the field of transplantation.

Perioperative care of organ transplant candidates or recipients is an exceedingly complex and multifaceted undertaking. It comprises three main components.

Pre-operative care includes the selection of the proper candidate. At this stage, the patient's medical and surgical history, current disease status, treatment progress, success or lack thereof and compliance with numerous medication regimes, are reviewed. The critical portion of the selection includes a current functional status assessment, the ability to tolerate multiple challenges of organ transplant surgery including that experienced during the post-operative period, and, most importantly, the prediction of outcome in both the immediate and long-term.

The degree of functional capacity impairment is a matter of continuous re-assessment and optimization in preparation for organ transplantation surgery.

Organ transplant surgery is a culmination of the transplantation process. The possibility of transplant surgery is contingent on availability, often immediate, and the proper quality of the donor organ. Intraoperative care for the organ transplant recipient, even in the relatively straightforward cases, is by far one of 
the most challenging tasks the transplant team encounters. The spectrum of problems and challenges include significant, sometimes life-threatening haemodynamic disturbances and acid-base /electrolytes dis-balance, significant ongoing blood loss, massive blood product administration, coagulation deficit correction and the necessity of temporary organ replacement techniques such as intra-operative dialysis.

Some of the most challenging aspects of intra-operative care for transplant recipient include the unpredictability of the timing, and the length of procedures, increased incidence of unanticipated complications, such as stress cardiomyopathy or intra-operative myocardial infarction.

Immediate post-operative care. The challenges here, albeit quite similar to those encountered in the operating room, are different in many ways. The reasons of major morbidity and mortality include a variety of cardiovascular complications, primary transplanted organ dysfunction or non-function, super-acute rejection, and numerous surgery-specific complications such as haemorrhage, vascular thrombosis, dehiscence, vascular and biliary anastomosis leaks, wound infections and sepsis $[3,4]$.

Also, seemingly trivial problems, such as haemodynamic instability, blood glucose fluctuations, acid-base disturbances, ventilator-associated problems, pulmonary complications and early cognitive dysfunctions, all require appropriate and immediate attention as well as the unremitting efforts of all care teams.

A deep understanding and detailed knowledge of each one of these components, their mutual influences, connections and interactions is a condition of paramount importance for any further progress in this field, in both scientific and practical aspects.

The current practice of immediate post-operative care for liver transplant patients encompasses a variety of pathways. For more than a decade, fast-track anaesthesia involving " in-the-operating room tracheal extubation" has been introduced and established as a safe practice for selected liver transplant recipients [5]. Today, many centres have adopted this practice, which brings substantial logistic and financial gains [6]. However, high-volume centres with ailing, high-MELD score recipients, continue to practice prolonged postoperative ventilation post-LT, with patients allocated to critical care units.
Historically, at least some of the recently transplanted cases ended up in the so-called pulmonary intensive care units, now termed medical intensive care units, where the emphasis is on prolonged mechanical ventilation management and associated issues. With the advent of ever-increasing numbers and complexity of transplant cases, and the establishment and rapid spreading of transplant programs both in Europe and in the US and Canada, it has become clear, that the capabilities of these units, and approach to post-transplant patients care were questionable. More recently, the concept of surgical ICUs has been almost universally adopted, and most, if not all, post-transplant patients are now admitted to such units.

Statistics indicated an improvement in immediate outcome and complications measures and for a time, SICUs became the placement of choice for posttransplant patients. Over, in the last ten years or so, it is becoming increasingly clear that these units, run by general ICU physicians and surgeons, have reached the pinnacle of their abilities to provide top-notch care for post-transplant patients. It has been observed, that many issues, specific for the transplant recipient, remain either underestimated and insufficiently addressed, or merely unrecognized, and, as a result, overlooked. Those specific issues have a high potential of organ and life-threatening complications, if not adequately addressed. To properly address these issues and problems, a paradigm shift is necessary, and some cultural changes are called upon. The necessity of highly specialized units, designed for post-operative care of organ transplant recipients is becoming apparent. ICU Physicians and nursing staff, working in such units, need specialized training in many specific areas pertinent to organ transplantation. The possible recourse, at least for the transitional period, would be involving transplant anaesthesia team members. Transplant anaesthesiologists, ideally with ICU fellowship training, are, most certainly, well-equipped physicians, capable of handling the majority of problems related to posttransplant patients. Such integration may prove fruitful in many ways, especially in the first 24-48 hours of ICU stay, when early complications are most common.

Of course, integration of other service members into the realm of critical care involves resolving many specific issues, such as logistics, funding and proper monetary compensation, scheduling working hours, staffing shifts, and also topics related to team responsibilities, allocating duties, establishing a chain of command, and 
many more. However, the idea of integrated critical care units, designed for and dedicated to the care of the sickest, most complex post-transplant patients, where physicians of different specialities work together, might prove beneficial over time.

And for the time being - it is undoubtedly worth consideration.

\section{- CONFLICT OF INTEREST}

None to declare

\section{REFERENCES}

1. Mar C, Álvarez M, Marco J, Mahíllo B, Gil D., et all. Global Organ Transplant Activities in 2015. Data from the Global Observatory on Donation and Transplantation (GODT). Abstracts, S29. Transplantation: August 2017. doi: 10.1097/01. tp.0000525015.43613.75
2. Transplants By Organ Type January 1, 1988 - April 30, 2019 Based on OPTN data as of May 22, 2019 https://optn. transplant.hrsa.gov/data/view-data-reports; Data repots WHO Global Observatory on Donation and Transplantation (GODT) www.transplant-observatory.org.

3. Fukazawa K, Pretto EA Jr, Nishida S, Reyes JD, Gologorsky EJ Factors associated with mortality within $24 \mathrm{~h}$ of liver transplantation: An updated analysis of 65,308 adult liver transplant recipients between 2002 and 2013.Clin Anesth. 2018;44:35-40.

4. Gopal PB, Kapoor D, Raya R, Subrahmanyam M, Juneja D, Sukanya B. Critical care issues in adult liver transplantation. Indian J Crit Care Med. 2009; 13(3):113-9.

5. Mandell MS, Stoner TJ, Barnett R, et al. A multicenter evaluation of safety of early extubation in liver transplant recipients.Liver Transpl. 2007;13(11):1557-63.

6. Bulatao IG, Heckman MG, Rawal B, et al. Avoiding stay in the intensive care unit after liver transplantation: A score to assign location of care. American J Transplant. 2014;14:2088-96 